\title{
Mobile terminal 3D image reconstruction program development based on Android Lin Qinhua
}

\author{
Sichuan Information Technology College $\quad 628017$
}

Keywords: 3D image reconstruction; curve approximation; matching constraint;

\begin{abstract}
During the study process of mobile terminal three-dimensional reconstruction program development based on Android, due to the adoption of the current algorithm for 3D image reconstruction program development, the definition of established model is low, leading to the problem of poor fidelity for 3D image reconstruction. Therefore, an Android mobile terminal 3D image reconstruction program development method based on improved space curve approximation algorithm is proposed. Under Android system architecture, the proposed method is based on triangulation measurement principle to calculate and obtain position deviation between image pixels, curve small region matching feature constraint thought is adopted to constrain features of 3D modeling, within a small feature space, through collision relation between scattered image feature points to set up precise compensation characteristics of 3D image reconstruction, thus greatly enhance the authenticity of 3D image reconstruction, and effectively complete mobile terminal 3D image reconstruction program development under the framework of Android system. Simulation results show that, Android mobile terminal 3D image reconstruction program development method based on improved space curve approximation algorithm can meet the application requirements of mobile terminal for 3D image reconstruction program development.
\end{abstract}

\section{Introduction}

In China, with the development of computer and network application popularization, the mobile device has become the main way for people to acquire information in daily life and work, and at the same time, the demand for mobile terminal also grow with each passing day (1). Mobile terminal 3D image reconstruction program development technology on the Android platform help users to get more rich and colorful services via the Internet, and satisfy the urgent needs of the user to access Internet whenever and wherever for entertainment, which attract the attention of many experts and scholars (2). Since it has a broad development space in the Android platform for mobile terminal 3D image reconstruction program development technology, has become the focus topic being researched in the industry, and has been widespread concerned, also a lot of good method are discovered $(3,4,5,6)$.

To solve the above problem, this paper proposes an Android mobile terminal 3D image reconstruction application development method based on improved spatial curve approximation algorithm.

\section{Principle of Android-based mobile terminal 3D image reconstruction program development method}

2.1 Feature constraint process of 3D modeling

In the optimizing process of mobile terminal 3D image reconstruction program development under the framework of Android system, $f_{n}(x, y)$ represents known image 2-D coordinates model, $f(x, y)$ is actual image 2-D coordinates, determining the similarity between two parameters, then $f_{n}(x, y)=f(x, y)$. The expression in the 3D image reconstruction program development optimization process, can reflect the gray value of some point in image template is same to gray value of corresponding value in real images, and a large number of interfering factors exist in the 
real image, resulting in $f_{n}(x, y)$ and $f(x, y)$ are unable to achieve the matching, thus, a certain error will be produced, based on triangulation principle, through the following formula to calculate corresponding image matching error:

$$
\begin{aligned}
& \omega(x, y)=\frac{\partial f}{\partial x} d x+\frac{\partial f}{\partial y} d y-\Delta f \\
& =f_{n}(x, y)-f(x, y)
\end{aligned}
$$

In the formula, $\frac{\partial f}{\partial x}$ represents the derivative of pixel gray in the $x$ direction, $\frac{\partial f}{\partial y}$ represents the derivative of pixel gray in the ${ }^{y}$ direction. During the process of optimization of mobile terminal 3D image reconstruction program development, curve matching constraint is used to approximate feature constraint process of three-dimensional modeling as below:

(1) epipolar constraint of curve segment.

(2) Uniqueness constraint.

(3) Attributes and gray similarity constraint.

In the mobile terminal 3D image reconstruction program development optimization process, based on similarity of curve characteristics can analyze using the matching degree of curve, described as below:

$$
\operatorname{SIM}\left[F N\left(H_{H}, H_{U}\right)\right]=\frac{\min \left(F N\left(H_{H}\right), F N\left(H_{U}\right)\right)}{\max \left(F N\left(H_{H}\right), F N\left(H_{U}\right)\right)}
$$

2.2 The implementation of mobile terminal 3D image reconstruction program development optimization method based on Android

Under Android system architecture, during the optimization process of mobil terminal 3D image reconstruction program, curve small region matching feature constraint thought is adopted to constrain features of 3D modeling, within a small feature space, through collision relation between scattered image feature points to set up precise compensation characteristics of 3D image reconstruction, thus greatly enhance the authenticity of 3D image reconstruction, and effectively complete mobile terminal 3D image reconstruction program development under the framework of Android system. The specific steps are detailed:

Assuming during the optimization process, the center coordinates location of the 3D image is determined by the following formula:

$$
c_{i}=\left[\begin{array}{l}
a_{i} \\
b_{i}
\end{array}\right]=\sum_{j=1}^{p} \lambda_{i j}\left[\begin{array}{l}
u_{i j} \\
v_{i j}
\end{array}\right]
$$

Among them, $c_{i}$ is the sum of $i$-th image of spatial position parameters and weights of all key characteristics, $\lambda_{i j}$ is the noise present in the original image and outlier disturbance parameters. The the mean variance algorithm can be used to obtain the value of interference weight parameters. Only for the direction component deviation $\sigma_{i j r}^{2}$ of all key details characteristics to process noise reduction.

The following formula is used to calculate the sum of the mean variance parameters $Q_{i j}$ of characteristic image:

$$
\sigma_{i j r}^{2}=\sigma_{i j 1}^{2}+\sigma_{i j 2}^{2}
$$

The value of $\lambda_{i j}$ is able to be obtained through operation: 


$$
\lambda_{i j}=\left(\sum_{j=1}^{p} \frac{\sum_{j=1}^{p}\left(\sigma_{i j 1}^{2}+\sigma_{i j 2}^{2}\right)}{\sum_{j=1}^{p}\left(\sigma_{l j 1}^{2}+\sigma_{l j 2}^{2}\right)}\right)^{-1}
$$

Through the above formula can be learned, weight coefficient and direction components variance parameters $\sigma_{i j 1}^{2}$ and $\sigma_{i j 2}^{2}$ are related. In the optimization process, after determining the center, in order to restore the normal three-dimensional image features, the relationship between the feature points need to be established, the space position relations of $\underline{X}_{F} \cdot \underline{n}$ and $\underline{X}_{D} \cdot \underline{n}$ is used, described as follow:

$$
\begin{array}{r}
\left\{\begin{array}{l}
\underline{X}_{F}=\underline{X}_{F}-\underline{A}_{k} \\
\underline{X}_{D}=\underline{D}_{K+1}-\underline{A}_{k}
\end{array}\right. \\
\left\{\begin{array}{l}
\underline{X}_{F}^{n}=\left(\underline{X}_{F} \cdot \underline{n}\right) \underline{n} \\
\underline{X}_{D}=\left(\underline{X}_{p} \cdot \underline{n}\right) \underline{n}
\end{array}\right.
\end{array}
$$

In the formula, $\underline{X}^{n}$ is the direction vector of $\underline{X}_{F}$, if the value sign of two 3D image feature data is inconsistent, then the line composed of point $D_{K}$ and $D_{K+1}$ and $\Delta A_{K} B_{K} C_{K}$ intersect or tangent.

Assuming the coordinate of 3D image collision point is $(x, y), Z$ value of corresponding coordinate to calculate plane collision transform function:

$$
z=f(x, y)=k e^{-\left(\frac{x-a}{m}\right)} e^{-\left(\frac{x-a}{m}\right)}
$$

In the formula, $k$ is the number of shape base, $a$ is the estimation error in the horizontal direction, $b$ is the estimation error in the longitudinal direction, $m$ represents the 3D image key features point distribution and the position in three-dimensional space, represented by the formula:

$$
p_{i}\left(x_{i}, y_{i}, z_{i}\right), i=1,2,3
$$

The above formula is used for logarithmic transformation, and then 3D image feature recovery operation is conducted, the following formula can be obtained:

$$
\left\{\begin{array}{c}
a=\frac{\left(y_{2}-y_{3}\right)\left[x_{1}^{2}-x_{2}^{2}+y_{1}^{2}-y_{2}^{2}+2\left(1 n z_{1}-1 n z_{2}\right)\right]}{2\left[\left(x_{1}-x_{2}\right)\left(y_{2}-y_{3}\right)-\left(x_{2}-x_{3}\right)\right]} \\
b=\frac{\left(x_{2}-x_{3}\right)\left[x_{1}^{2}-x_{2}^{2}+y_{1}^{2}-y_{2}^{2}+2\left(1 n z_{1}-1 n z_{2}\right) m^{2}\right]}{-2\left[\left(x_{1}-x_{2}\right)\left(y_{2}-y_{3}\right)-\left(x_{2}-x_{3}\right)\right]} \\
z_{1}=k e^{-\left(\frac{x-a}{m}\right)} e^{-\left(\frac{x-a}{m}\right)} \\
z=f(x, y)=k e^{-\left(\frac{x-a}{m}\right)} e^{-\left(\frac{x-a}{m}\right)}
\end{array}\right.
$$

Through the above stated method, in the Android system, precise compensation characteristics of 3D image reconstruction is established, thus greatly enhance the authenticity of 3D image reconstruction, and effectively complete mobile terminal 3D image reconstruction program development under the framework of Android system.

\section{3 simulation experiments}

In order to prove that the effectiveness of Android mobile terminal 3D image reconstruction 
program development method based on improved spatial curve approximation algorithm, there is the need for an experiment, experiment operation environment is: Matlab6.O, windows operating system, CPU, , 2 Dual Core Pentium 2G memory. Experiment samples were chosen from a group of image test data from mobile terminal 3D image reconstruction system under Android system framework, the traditional method and Android mobile terminal 3D image reconstruction program development method based on the improved space curve approximation algorithm were adopted. Two different algorithms were utilized in experiments for mobile terminal 3D image reconstruction measurement in the Android system, the results are shown in table 1.

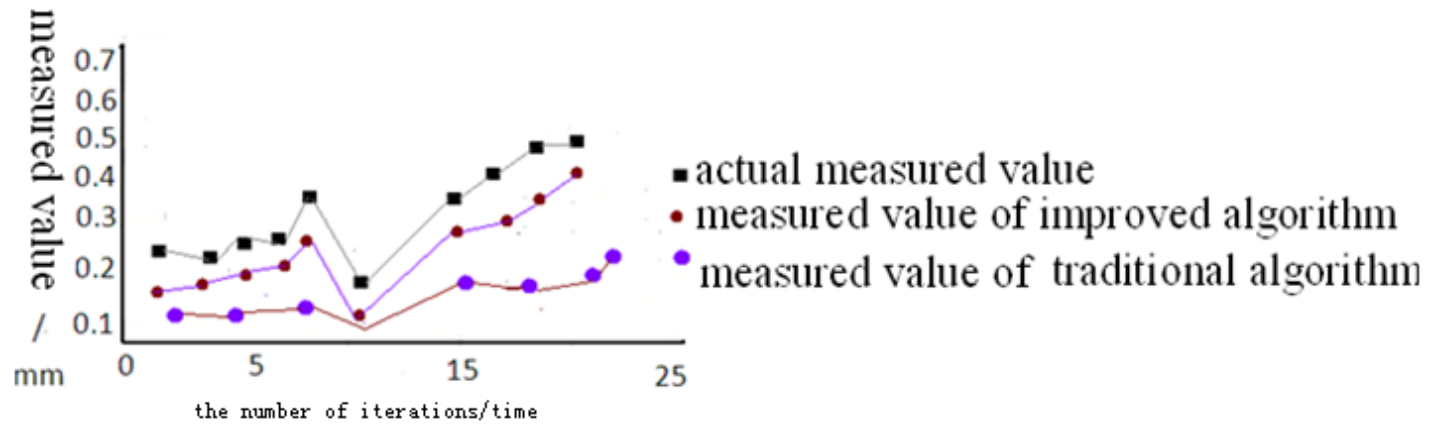

Fig. 1 Comparison of measured values obtained with different algorithms

It can be seen from Fig.1, the measured value of proposed algorithm and actual measured value have better matching and higher accuracy. Since under Android system architecture, the proposed method is based on triangulation measurement principle to calculate and obtain position deviation between image pixels, curve small region matching feature constraint thought is adopted to constrain features of 3D modeling, within a small feature space, through collision relation between scattered image feature points to set up precise compensation characteristics of 3D image reconstruction, thus greatly enhance the authenticity of 3D image reconstruction. While the measured value of traditional algorithm has a big difference from actual value, results in significant deviation.

The experiment can prove that the Android mobile terminal 3D image reconstruction program development method based on improved spatial curve approximation algorithm has high precision and strong practicability.

\section{Conclusions}

With the adoption of the current algorithm for 3D image reconstruction program development, the definition of established model is low, leading to the problem of poor fidelity for 3D image reconstruction. Therefore, an Android mobile terminal 3D image reconstruction program development method based on improved space curve approximation algorithm is proposed. Under Android system architecture, the proposed method is based on triangulation measurement principle to calculate and obtain position deviation between image pixels, curve small region matching feature constraint thought is adopted to constrain features of 3D modeling, within a small feature space, through collision relation between scattered image feature points to set up precise compensation characteristics of 3D image reconstruction, thus greatly enhance the authenticity of 3D image reconstruction, and effectively complete mobile terminal 3D image reconstruction program development under the framework of Android system. Simulation results show that, Android mobile terminal 3D image reconstruction program development method based on improved space curve approximation algorithm can meet the application requirements of mobile terminal for 3D image reconstruction program development.

\section{References}

[1] Fan Zheyi, Zhou Zhiguo, Liu Zhiwen, Pan Limin, He Bingsong. Image tracking experiment system based on TMS320DM642 and modelling design [J]. Experimental technology and management.2013.3:74-77. 
[2] Liu Xue, Jing Wenbo, Wang Xiaoman. A guidance tracking algorithm for visible light image of air to ground [J]. Journal of Changchun University of Science and Technology: Natural Science, 2014.5:87-90.

[3] Li Shuiping, Wu Yu. Fast implementation method of 3D image reconstruction based on texture fusion [J]. Computer technology and development. 2014.5:138-141.

[4] Wei Chuliang, Luo Peiyu, Hong Xiaobin, Qiu Chaole, Cai Zemin. Study on a new method for 3D image reconstruction based on single photograph mode [J]. Chinese test.2014.2:23-26.

[5] Chen Deyun, Gao Ming, Song Leilin, Jia Nan, Yu Xiaoyang. A new 3D ECT sensor and model 3D image reconstruction method [J]. Chinese Journal of scientific instrument, 2014.5:961-968.

[6] Zhang Xiaojuan. Research of difference information fusion algorithm in three dimensional image reconstruction [J]. Bulletin of science and technology.2014.12:41-42. 